\title{
Comparison of the optical quality after SMILE and FS-LASIK for high myopia by OQAS and iTrace analyzer: a one-year retrospective study
}

Yewei Yin ${ }^{1,2}$, Ying $\mathrm{Lu}^{1}$, Aiqun Xiang ${ }^{1}$, Yanyan $\mathrm{Fu}^{1}$, Yang Zhao ${ }^{1}$, Yuanjun $\mathrm{Li}^{1,2}, \mathrm{Tu} \mathrm{Hu}^{1,2}$, Kaixuan Du ${ }^{1,2}$, Shengfa $\mathrm{Hu}^{1,2}$, Qiuman Fu', Xiaoying $\mathrm{Wu}^{1,2}$ and Dan Wen ${ }^{1,2^{*}}$

\begin{abstract}
Background: To compare the correction effect and optical quality after small-incision lenticule extraction (SMILE) and femtosecond laser assisted laser in situ keratomileusis (FS-LASIK) for high myopia.

Methods: 51 high myopia eyes after SMILE and 49 high myopia eyes after FS-LASIK were enrolled and divided into two groups retrospectively. The OQAS and iTrace analyzer were used for optical quality inspection. Between the two groups the spherical equivalent (SE), astigmatism, uncorrected distant visual acuity (UDVA), strehl ratio (SR), modulation transfer function cutoff frequency (MTF cutoff), objective scatter index (OSI) and wavefront aberrations were analyzed and compared before surgery and at 1, 6 and 12 months after surgery.

Results: After the operation: (1) SE and astigmatism declined and UDVA increased significantly in both groups, and UDVA was better after SMILE than FS-LASIK. (2) SR and MTF cutoff reduced and OSI increased significantly after SMILE and FS-LASIK. SR and MTF cutoff were significantly higher after SMILE than FS-LASIK. OSI was significantly lower after SMILE than FS-LASIK. (3) The total wavefront aberration, total low-order wavefront aberration, defocus and astigmatism aberration as well as trefoil aberration reduced significantly in both groups. The total high-order wavefront aberration increased significantly after FS-LASIK. The spherical and coma aberration increased significantly in both groups. The total high-order wavefront aberration and coma aberration at 1 month were higher after FSLASIK than SMILE.

Conclusion: The optical quality descended after SMILE and FS-LASIK. SMILE was superior to FS-LASIK at the correction effect and optical quality for high myopia. The combination of OQAS and iTrace analyzer is a valuable complementary measurement in evaluating the optical quality after the refractive surgery.
\end{abstract}

Trial registration: This is a retrospective study. This research was approved by the ethics committee of Xiangya Hospital and the IRB approval number is 201612074.

Keywords: SMILE, FS-LASIK, High myopia, OQAS, iTrace, Optical quality

\footnotetext{
* Correspondence: wendan@csu.edu.cn

'Eye Center of Xiangya Hospital, Central South University, Changsha 410008, Hunan, China

${ }^{2}$ Hunan Key Laboratory of Ophthalmology, Changsha 410008, Hunan, China
}

C C The Author(s). 2021 Open Access This article is licensed under a Creative Commons Attribution 4.0 International License, which permits use, sharing, adaptation, distribution and reproduction in any medium or format, as long as you give appropriate credit to the original author(s) and the source, provide a link to the Creative Commons licence, and indicate if changes were made. The images or other third party material in this article are included in the article's Creative Commons licence, unless indicated otherwise in a credit line to the material. If material is not included in the article's Creative Commons licence and your intended use is not permitted by statutory regulation or exceeds the permitted use, you will need to obtain permission directly from the copyright holder. To view a copy of this licence, visit http://creativecommons.org/licenses/by/4.0/. The Creative Commons Public Domain Dedication waiver (http://creativecommons.org/publicdomain/zero/1.0/) applies to the data made available in this article, unless otherwise stated in a credit line to the data. 


\section{Background}

Being a common eye ametropia disease, high myopia has now widely existed in the general population and its incidence rate still keeps increasing so far. Meanwhile, the refractive surgery develops quite quickly in recent years and increasing numbers of patients with high myopia choose the surgery to correct the refractive errors $[1,2]$. In the field of corneal refractive surgery, both smallincision lenticule extraction (SMILE) and femtosecond laser assisted laser in situ keratomileusis (FS-LASIK) have been extensively applied [3, 4]. When performing the SMILE surgery, a corneal stromal lenticule is firstly made with the femtosecond laser and then extracted from a small side incision [5]. As a comparison, femtosecond laser is utilized in the FS-LASIK surgery to generate the corneal flap, and excimer laser is applied to ablate the corneal stroma subsequently [6].

In order to effectively evaluate the effect after the refractive surgery, different kinds of examination methods and evaluating indicators were used in previous work [7-9]. Thereinto, the optical quality is believed to be the most important indicator [10]. According to most of clinical data, the optical quality after SMILE is generally better than that after FS-LASIK [11-13]. However, most of these data are obtained from the patients with low and moderate myopia. Due to the high refractive error and limited corneal thickness, the corneal refractive surgery could not be widely performed for the patients with high myopia. Few observations about the optical quality after SMILE and FS-LASIK were done for the high myopic patients. Therefore, it is meaningful to evaluate the optical quality objectively in high myopia correction after SMILE and FS-LASIK.

A comprehensive measurement of multiple indicators is needed for the effective evaluation of the postoperative optical quality. Considering the existing visual quality inspection equipments, both the Optical Quality Analysis System (OQAS, Visiometrics, Terrassa, Spain) and Tracey-iTrace Visual Function Analyzer (iTrace, Texas, USA) could offer an effective and valid method to evaluate the effectiveness and outcomes of corneal refractive surgery [14-16]. In this work, we intended to obtain the objective data of the optical quality for the patients with high myopia and compare the optical quality after SMILE and FS-LASIK by simultaneously applying the OQAS and iTrace analyzer.

\section{Patients and methods}

\section{Patients and study design}

In this retrospective study, the clinical data of the right eyes of 100 patients with high myopia were collected and analyzed. Thereinto, 51 eyes (27 females, 24 males) had the SMILE surgery (hereinafter called SMILE group) and 49 eyes (26 females, 23 males) had FS-LASIK (hereinafter called FS-LASIK group) after considering comprehensively the corneal thickness, refractive errors to be corrected and the personal requirements. The operation date and follow-up for these patients was from November 1st, 2017 to November 31st, 2020 in the Eye Center of Xiangya Hospital, Central South University, China. The inclusion criteria of enrolling these patients was as follows: (1) the age was 18 years old or older, (2) the spherical refraction was from $-6.00 \mathrm{D}$ to $-10.00 \mathrm{D}$ and the astigmatism was from $0 \mathrm{D}$ to $-2.50 \mathrm{D}$, (3) there was no history of other eye diseases that might impair the patients' vision, (4) the follow-up duration was more than one year with regular check at 1, 6 and 12 months postoperatively, (5) both the SMILE and FS-LASIK surgery were performed by the same experienced surgeon and the examinations before and after operation were performed by the same experienced technicians.

\section{Surgical procedures}

For the SMILE surgery, the VisuMax $500 \mathrm{kHz}$ femtosecond laser (Carl Zeiss Meditec AG, Germany) was applied to create the corneal stromal lenticule and side incision. The laser energy was 130nJ, and the spacing between the points and rows was both $4.5 \mu \mathrm{m}$. The diameter of the lenticules (i.e. programmed optical zone) was $6.0 \sim$ $6.8 \mathrm{~mm}$, and the corneal cap thickness was $100 \sim$ $120 \mu \mathrm{m}$. The side-cut angle was $90^{\circ}$ with a circumferential width of $4.0 \mathrm{~mm}$.

As to the FS-LASIK surgery, the corneal flap was fabricated with VisuMax $500 \mathrm{kHz}$ femtosecond laser with energy of $185 \mathrm{~nJ}$. The thickness of the corneal flap was $90 \mu \mathrm{m}$, and the diameter of the flap was $8.0 \mathrm{~mm}$ with standard $90^{\circ}$ hinge and $90^{\circ}$ side-cut angle. The ablation on the corneal stroma was accomplished with the excimer laser (VISX Star S4 Custom VSS Excimer Laser System, America) with a repetition rate of $250 \mathrm{~Hz}$.

In the SMILE and FS-LASIK surgery the treatment center was the corneal vertex and no particular adjustments were performed to the manufacturer's nomograms. Postoperatively, the treatment regimen was identical for both groups, including tobramycin dexamethasone (Alcon Couvreur, Belgium) four times daily in the first week, $0.3 \%$ tobramycin (Alcon Couvreur, Belgium) and 0.1\% fluorometholone (Santen, Japan) four times daily in the second week, $0.1 \%$ fluorometholone (Santen, Japan) and pranoprofen (Senju, Japan) four times daily in the third week, and pranoprofen (Senju, Japan) four times daily in the fourth week, and $0.1 \%$ hyaluronic acid sodium (Ursapharm Arzneimittel GmbH, Germany) four times daily in a month.

\section{Examinations}

The examinations were performed before surgery and at $1,6,12$ months after surgery, including the slit-lamp 
examination, the eyesight test using the standard logarithmic visual acuity chart and snellen visual acuity chart, the manifest refraction, the OQAS examination and iTrace analyzer examination. The OQAS was applied with $4.0 \mathrm{~mm}$ pupil diameter to get the parameters including strehl ratio (SR), modulation transfer function cutoff frequency (MTF cutoff, c/deg) and objective scatter index (OSI). The iTrace analyzer was used with 4.0 $\mathrm{mm}$ pupil diameter to get the data of the total ocular wavefront aberrations, including the total wavefront aberration (TWA), total low-order wavefront aberration (TLOA), total high-order wavefront aberration (THOA), defocus aberration (DA), astigmatism aberration (AA), spherical aberration (SA), coma aberration (CA) and trefoil aberration (TA). The examinations were completed by the same technicians respectively.

\section{Statistical analyses}

The SPSS statistical package (Version 25.0; IBM SPSS Inc., Chicago, Illinois, USA) and the Microsoft Excel Software were applied for the statistical analysis. The one-way repetitive measurement and analysis of variance was applied to analyze the difference of spherical equivalent (SE), astigmatism, uncorrected distant visual acuity (UDVA), SR, MTF cutoff, OSI and wavefront aberrations before surgery and at 1, 6 and 12 months after surgery when the data followed the law of normal distribution. Otherwise, the Friedman test was used. The paired $t$-test was used to analyze the difference of age, SE, astigmatism, UDVA, SR, MTF cutoff, OSI and wavefront aberrations at each point of time between the two groups. And the multiple-factor repetitive measurement and analysis of variance was used to compare the pre and post-operative variation of SE, astigmatism, UDVA, SR, MTF cutoff, OSI and wavefront aberrations between the SMILE and FS-LASIK group. $p<0.05$ was taken as being statistically significant, and the adjusted alpha i.e., alpha/no. of test, was adopted for the analysis with repeated measurement.

\section{Results}

Descriptive statistics of the patients, UDVA and the other refractive outcomes

No intraoperative or postoperative complications, such as corneal infection, haze, corneal epithelial ingrowth and elevated intraocular pressure, occurred in both the SMILE and FS-LASIK group. As summarized in Table 1, there were no significant differences between the two groups in terms of age, preoperative and postoperative $\mathrm{SE}$ and astigmatism. Both SE and astigmatism significantly decreased after SMILE and FS-LASIK $(p<0.05)$. Between the two groups there were no significant differences in SE and astigmatism at each point of time, and no significant differences in SE and astigmatism's variation before and after the operation were found $\left(p^{\mathrm{SE}}=\right.$ $0.119, p^{\text {astig }}=0.782$ ). The preoperative UDVA had no significant difference between the two groups and increased significantly after operation until the 12th

Table 1 The preoperative and postoperative demographics (mean \pm SD) for the SMILE and FS-LASIK group

\begin{tabular}{|c|c|c|c|c|}
\hline Parameters & & SMILE group & FS-LASIK group & $p_{1}$-value \\
\hline Age (years old) & & $23.9 \pm 4.8$ & $24.4 \pm 4.9$ & 0.605 \\
\hline \multirow[t]{5}{*}{ SE(D) } & Preop & $-7.964 \pm 0.943$ & $-8.390 \pm 1.372$ & 0.078 \\
\hline & Post 1mo & $-0.194 \pm 0.549^{*}$ & $-0.230 \pm 0.487^{*}$ & 0.740 \\
\hline & Post $6 \mathrm{mo}$ & $-0.156 \pm 0.486^{*}$ & $-0.194 \pm 0.459^{*}$ & 0.677 \\
\hline & Post $12 \mathrm{mo}$ & $-0.204 \pm 0.461^{*}$ & $-0.334 \pm 0.441^{*}$ & 0.169 \\
\hline & $p_{0}$-value & 0.119 & & \\
\hline \multirow[t]{5}{*}{ Astigmatism (D) } & Preop & $-0.786 \pm 0.508$ & $-0.806 \pm 0.736$ & 0.846 \\
\hline & Post $1 \mathrm{mo}$ & $0.005 \pm 0.377^{*}$ & $-0.031 \pm 0.453^{*}$ & 0.655 \\
\hline & Post $6 \mathrm{mo}$ & $-0.163 \pm 0.321^{*}$ & $-0.102 \pm 0.398^{*}$ & 0.326 \\
\hline & Post $12 \mathrm{mo}$ & $-0.046 \pm 0.356^{*}$ & $-0.031 \pm 0.352^{*}$ & 0.832 \\
\hline & $p_{0}$-value & 0.782 & & \\
\hline \multirow[t]{5}{*}{ UDVA (LogMAR) } & Preop & $-1.292 \pm 0.274$ & $-1.335 \pm 0.289$ & 0.427 \\
\hline & Post 1mo & $0.129 \pm 0.167^{*}$ & $0.025 \pm 0.111^{*}$ & $<0.001$ \\
\hline & Post $6 \mathrm{mo}$ & $0.104 \pm 0.110^{*}$ & $0.045 \pm 0.121^{*}$ & 0.023 \\
\hline & Post 12mo & $0.099 \pm 0.106^{*}$ & $0.032 \pm 0.176^{*}$ & 0.021 \\
\hline & $p_{0}$-value & 0.481 & & \\
\hline
\end{tabular}

SD: standard deviation. Preop: preoperative. Post $1 \mathrm{mo}$ : at 1 month postoperatively. Post $6 \mathrm{mo}$ : at 6 months postoperatively. Post $12 \mathrm{mo}$ : at 12 months postoperatively. $p_{0}=p$ value of the difference for the pre and post-operative variation of SE, astigmatism and UDVA between the SMILE and FS-LASIK group. $p_{1}=$ $p$ value of the difference in age, $\mathrm{SE}$, astigmatism and UDVA at each point of time between the SMILE and FS-LASIK group. When $p<0.05$, the difference was statistically significant. ${ }^{*}$ Compared with that before the operation, $p<$ adjusted alpha 
month in both groups $(p<0.05)$. The pre and postoperative variation of UDVA had no significant difference between the two groups $(p=0.481)$, and the postoperative UDVAs in the SMILE group were better than those in the FS-LASIK group with significant differences $(p<0.05)$.

Figure 1 shows the standard graphs for reporting the clinical results of the two corneal refractive surgeries. At 12 months after the operation, 92\% (47/51) of treated eyes in the SMILE group and 74\% (36/49) in the FSLASIK group had a postoperative snellen visual acuity of $20 / 20$ or better. And the corrected distant visual acuity (CDVA) of $12 \%$ of eyes in the SMILE group and $32 \%$ in the FS-LASIK group had 2 or more lines lost at 12 months postoperatively. 75 and $65 \%$ of eyes achieved postoperative SE within +/-0.50 D at 12 months after SMILE and FS-LASIK respectively. From the 1st to the 12 th month, $17 \%$ of eyes in the SMILE group and $14 \%$ in the FS-LASIK group had $>0.50 \mathrm{D}$ change of SE. At 12 months postoperatively, 95 and $100 \%$ of eyes had the refractive astigmatism less than or equal to $+/-0.50 \mathrm{D}$ in the SMILE and FS-LASIK group respectively.

\section{SR, MTF cutoff and OSI}

Preoperatively, there were no significant differences in SR, MTF cutoff and OSI between the SMILE and FSLASIK group $\left(p^{\mathrm{SR}}=0.259, p^{\mathrm{MTF}}\right.$ cutoff $=0.151, p^{\mathrm{OSI}}=$ 0.719). After the surgery, SR and MTF cutoff in the two groups both reduced significantly $(p<0.05)$ except the MTF cutoff at 12 months in the SMILE group ( $p=$ 0.055). Compared with those in the FS-LASIK group, the postoperative SR and MTF cutoff were obviously more in the SMILE group at each point of observing time $(p<0.05)$. The pre and post-operative variation of SR and MTF cutoff had no significant difference between the two groups $\left(p^{\mathrm{SR}}=0.057, p^{\mathrm{MTF}}\right.$ cutoff $\left.=0.062\right)$. On the other hand, OSI increased significantly after both surgeries $(p<0.05)$. The pre and post-operative variation of OSI showed significant difference between the two groups $(p<0.001)$. Moreover, after the operation OSI in the FS-LASIK group was much more than that in the SMILE group $(p<0.05)$ as summarized in Table 2.

\section{The wavefront aberrations}

As shown in Table 3, no significant differences in TWA, TLOA and THOA before the operation were found between the SMILE and FS-LASIK group. After the operation, both TWA and TLOA reduced significantly in the two groups $(p<0.05)$, and their pre and post-operative variation showed no significant difference between the two groups $\left(p^{\mathrm{TWA}}=0.115, p^{\mathrm{TLOA}}=0.061\right)$. There were no significant differences in postoperative TWAs and TLOAs at each point of time between the two groups. The THOAs did not change significantly after SMILE.
In contrast, THOA increased significantly after FSLASIK $(p<0.05)$ and was significantly more than that in the SMILE group at 1 month $(p=0.033)$. The pre and post-operative variation of THOA showed no significant difference between the two groups $(p=0.115)$.

The defocus aberration (DA) and astigmatism aberration (AA) both decreased significantly at 1,6 and 12 months after the two surgeries $(p<0.05)$. No significant differences for their pre and post-operative variations were found between the SMILE and FS-LASIK group $\left(p^{\mathrm{DA}}=0.085, p^{\mathrm{AA}}=0.119\right)$. Moreover, the DAs and AAs had no significant difference between the two groups, preoperatively and postoperatively. The spherical aberration (SA) and coma aberration (CA) increased significantly at 1, 6 and 12 months after the two surgeries and their pre and post-operative variation showed no significant difference between the two groups $\left(p^{\mathrm{SA}}=0.467\right.$, $p^{\mathrm{CA}}=0.216$ ). In addition, there were no significant differences in SAs and CAs between the two groups preoperatively and postoperatively except CA at 1 month which was more in FS-LASIK $(p=0.019)$. Furthermore, the trefoil aberration (TA) reduced significantly after the operation in both groups $(p<0.05)$ and its pre and postoperative variation had no significant difference between the two groups. It's also illustrated that there were no significant differences in TAs between the two groups preoperatively and postoperatively in Table 4.

\section{Discussion}

As the safest myopic surgeries in recent years, SMILE and FS-LASIK are widely applied for myopia correction. The theory and procedure are different between the two surgeries. But similarly, for high myopia correction, more corneal tissue will be removed in the two surgeries and the corneal refractive power is more likely to be instable. However, few studies have focused on the correction effect and optical quality after SMILE and FSLASIK in high myopia. Hence this study worked on evaluating and comparing the correction effect and optical quality after SMILE and FS-LASIK for patients with high myopia.

The visual acuity and refractive power are the most commonly used indicators to illustrate the effectiveness of myopic surgery. In this work, both SMILE and FSLASIK could effectively correct the refractive errors and improve the naked visual acuity of the patients with high myopia, similar with the other studies [17-19]. This study also found that the postoperative visual acuity (UDVA) and refractive results (SE and refractive astigmatism) after SMILE were better than those after FSLASIK. Hence one can see that SMILE had an advantage over FS-LASIK at achieving more satisfactory visual acuity and refractive results, at least for a year. It was noteworthy that a loss of visual acuity occurred at 12 months 

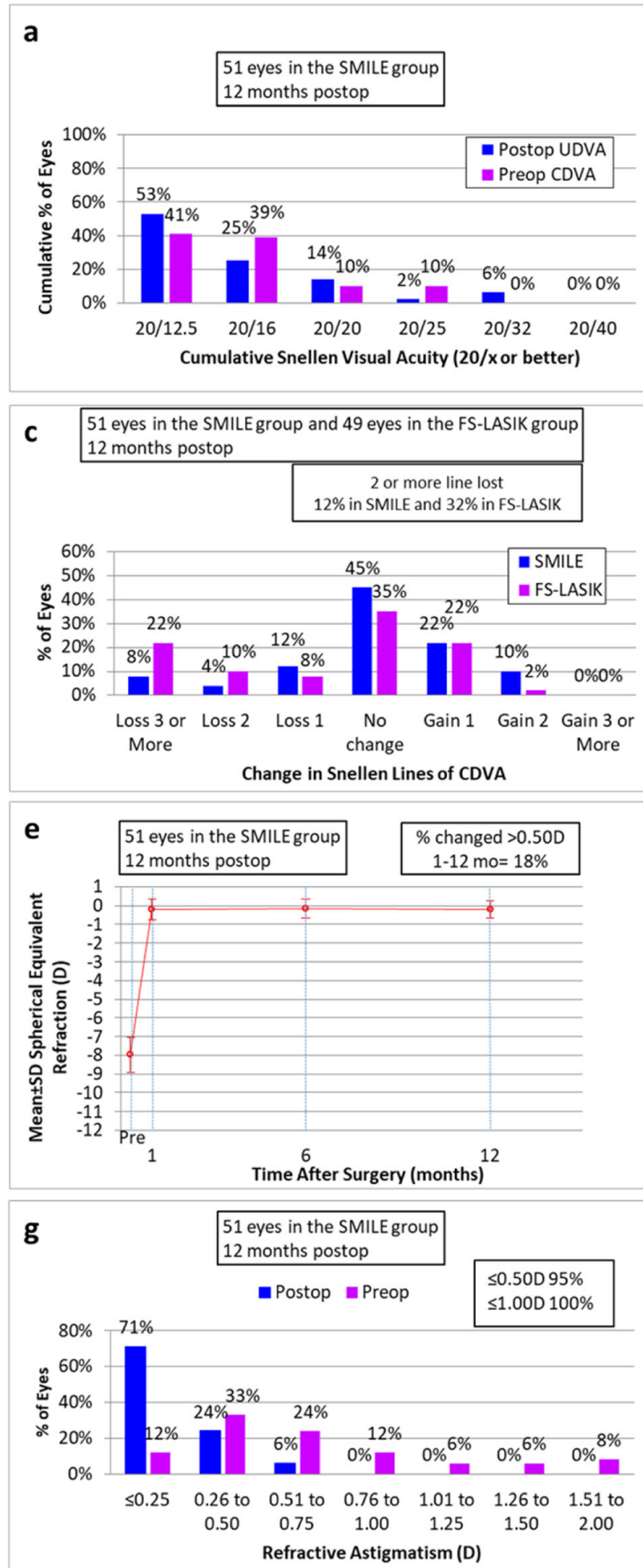

b


d 51 eyes in the SMILE group and 49 eyes in the FS-LASIK group 12 months postop

\section{-SMILE $=$ FS-LASIK}

$35 \%$
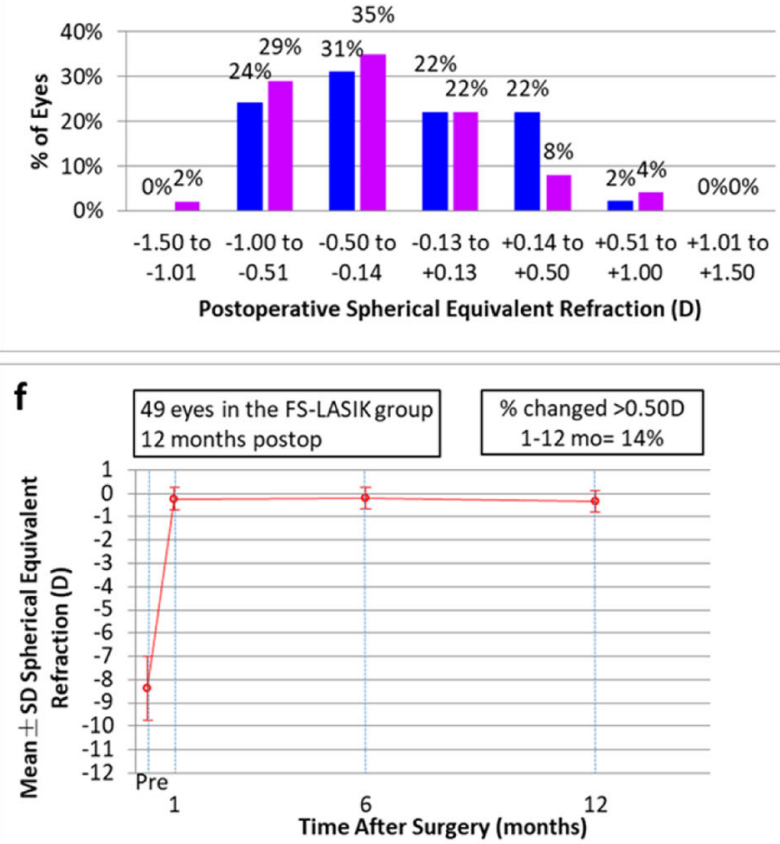

h

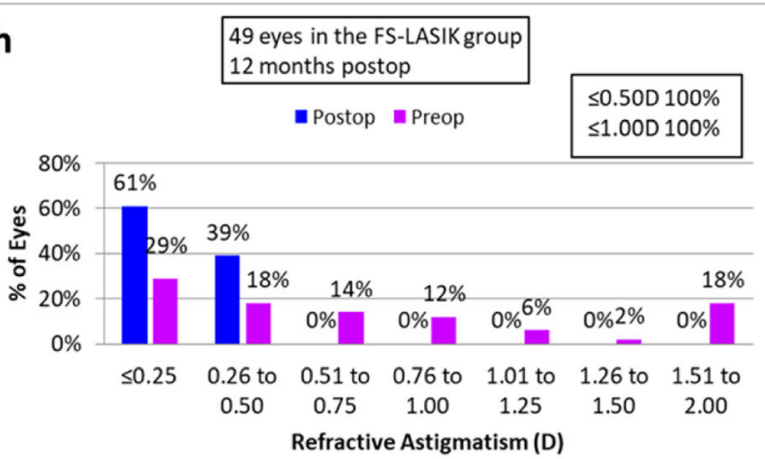

Fig. 1 The standard graphs for reporting the visual and refractive outcomes of 51 eyes in the SMILE group and 49 eyes in the FS-LASIK group at 12 months postoperatively 
Table 2 The variation of the SR, MTF cutoff and OSI with time in the SMILE and FS-LASIK group (mean \pm SD)

\begin{tabular}{|c|c|c|c|c|c|}
\hline & Preop & Post 1mo & Post $6 \mathrm{mo}$ & Post $12 \mathrm{mo}$ & $p_{0}$-value \\
\hline & $S R$ & & & & \\
\hline SMILE group & $0.260 \pm 0.064$ & $0.221 \pm 0.069^{*}$ & $0.212 \pm 0.057^{*}$ & $0.233 \pm 0.069^{*}$ & 0.057 \\
\hline FS-LASIK group & $0.245 \pm 0.077$ & $0.185 \pm 0.062^{*}$ & $0.175 \pm 0.062^{*}$ & $0.178 \pm 0.047^{*}$ & \\
\hline \multirow[t]{2}{*}{$p_{1}$-value } & 0.259 & 0.010 & 0.007 & $<0.001$ & \\
\hline & MTF cutoff & & & & \\
\hline SMILE group & $43.684 \pm 7.955$ & $39.161 \pm 10.711^{*}$ & $37.547 \pm 10.297^{*}$ & $40.552 \pm 10.005$ & 0.062 \\
\hline FS-LASIK group & $41.462 \pm 9.809$ & $32.520 \pm 12.305^{*}$ & $32.361 \pm 10.764^{*}$ & $31.489 \pm 9.626^{*}$ & \\
\hline \multirow[t]{2}{*}{$p_{1}$-value } & 0.151 & 0.007 & 0.029 & $<0.001$ & \\
\hline & OSI & & & & \\
\hline SMILE group & $0.49 \pm 0.24$ & $0.88 \pm 0.53^{*}$ & $0.84 \pm 0.44^{*}$ & $0.80 \pm 0.53^{*}$ & $<0.001$ \\
\hline FS-LASIK group & $0.59 \pm 0.33$ & $1.63 \pm 1.01^{*}$ & $1.39 \pm 0.92^{*}$ & $1.29 \pm 0.72^{*}$ & \\
\hline$p_{1}$-value & 0.719 & $<0.001$ & 0.001 & 0.001 & \\
\hline
\end{tabular}

SD: standard deviation. Preop: preoperative. Post $1 \mathrm{mo}$ : at 1 month postoperatively. Post $6 \mathrm{mo}$ : at 6 months postoperatively. Post $12 \mathrm{mo}$ : at 12 months postoperatively. $p_{0}=p$ value of the difference for the pre and post-operative variation of SR, MTF cutoff and OSI between the SMILE and FS-LASIK group. $p_{1}=p$ value of the difference in SR, MTF cutoff and OSI at each point of time between the SMILE and FS-LASIK group. When $p<0.05$, the difference was statistically significant. * Compared with that before the surgery, $p<$ adjusted alpha

after SMILE and FS-LASIK, consistent with the change of spherical equivalent refraction. We think the main reason for the loss is that the subjects are all high myopia patients, as it has been proved that the refractive regression is more likely to occur in patients with high myopia [20, 21]. We also analyzed the individual eyes with visual acuity loss and speculated that it might be the result of abnormal accommodation function before surgery in these young high myopia patients [22]. A previous research also concluded that a decrease in amplitude of accommodation and facility of accommodation might result in some of the near-vision problems in younger myopes in early postoperative days after refractive surgery [23]. Nevertheless, as we did not measure the accommodation function of all these subjects preoperatively, there is no hard proof for this assumption.

Due to the postoperative corneal deformation, the increased corneal scatter, wavefront aberrations and other factors might affect the optical quality [24-26]. Hence this study measured and evaluated the postoperative objective optical quality. Because of the limitation of a single detection equipment, this study used OQAS and iTrace analyzer simultaneously which were both widely applied for objective optical quality inspection and

Table 3 The variation of total wavefront aberrations with time in the SMILE and FS-LASIK group (mean \pm SD)

\begin{tabular}{lllll}
\hline & Preop & Post 1 mo & Post 6mo & Post 12mo \\
\hline & TWA & & & $\boldsymbol{p}_{\mathbf{0}}$-value \\
SMILE group & $7.896 \pm 2.842$ & $1.910 \pm 1.830^{*}$ & $2.014 \pm 1.665^{*}$ & $2.156 \pm 1.791^{*}$ \\
FS-LASIK group & $8.797 \pm 3.459$ & $1.965 \pm 1.582^{*}$ & $1.766 \pm 1.509^{*}$ & $2.376 \pm 1.761^{*}$ \\
$p_{1}$-value & 0.122 & 0.841 & 0.440 & 0.539 \\
& TLOA & & & 0.115 \\
SMILE group & $7.756 \pm 2.967$ & $1.812 \pm 1.778^{*}$ & $1.900 \pm 1.585^{*}$ & $2.052 \pm 1.687^{*}$ \\
FS-LASIK group & $8.780 \pm 3.446$ & $1.717 \pm 1.476^{*}$ & $1.572 \pm 1.444^{*}$ & $2.226 \pm 1.683^{*}$ \\
$p_{1}$-value & 0.099 & 0.783 & 0.293 & 0.623 \\
& THOA & & & 0.061 \\
SMILE group & $0.565 \pm 0.775$ & $0.543 \pm 0.512$ & $0.594 \pm 0.597$ & $0.598 \pm 0.665$ \\
FS-LASIK group & $0.485 \pm 0.384$ & $0.789 \pm 0.727^{*}$ & $0.714 \pm 0.575^{*}$ & $0.741 \pm 0.642^{*}$ \\
$p_{1}$-value & 0.514 & 0.033 & 0.307 & 0.251 \\
\hline
\end{tabular}

SD: standard deviation. Preop: preoperative. Post $1 \mathrm{mo}$ : at 1 month postoperatively. Post $6 \mathrm{mo}$ : at 6 months postoperatively. Post $12 \mathrm{mo}$ : at 12 months postoperatively. TWA: total wavefront aberration. TLOA: total low-order wavefront aberration. THOA: total high-order wavefront aberration. $p_{0}=p$ value of the difference for the pre and post-operative variation of TWA, TLOA and THOA between the SMILE and FS-LASIK group. $p_{1}=p$ value of the difference in TWA, TLOA and THOA at each point of time between the SMILE and FS-LASIK group. When $p<0.05$, the difference was statistically significant. * Compared with that before the surgery, $p<$ adjusted alpha 
Table 4 The variation of the wavefront aberrations with time in the SMILE and FS-LASIK group (mean \pm SD)

\begin{tabular}{|c|c|c|c|c|c|}
\hline & Preop & Post $1 \mathrm{mo}$ & Post $6 \mathrm{mo}$ & Post $12 \mathrm{mo}$ & $p_{0}$-value \\
\hline & $\mathrm{DA}$ & & & & \\
\hline SMILE group & $7.814 \pm 2.800$ & $1.714 \pm 1.797^{*}$ & $1.824 \pm 1.598^{*}$ & $1.972 \pm 1.670^{*}$ & 0.085 \\
\hline FS-LASIK group & $8.671 \pm 3.370$ & $1.651 \pm 1.458^{*}$ & $1.470 \pm 1.462^{*}$ & $2.152 \pm 1.704^{*}$ & \\
\hline \multirow[t]{2}{*}{$p_{1}$-value } & 0.133 & 0.856 & 0.250 & 0.613 & \\
\hline & $\mathrm{AA}$ & & & & \\
\hline SMILE group & $0.967 \pm 0.557$ & $0.381 \pm 0.357^{*}$ & $0.391 \pm 0.300^{*}$ & $0.441 \pm 0.431^{*}$ & 0.119 \\
\hline FS-LASIK group & $1.207 \pm 0.988$ & $0.420 \pm 0.434^{*}$ & $0.380 \pm 0.344^{*}$ & $0.427 \pm 0.350^{*}$ & \\
\hline \multirow[t]{2}{*}{$p_{1}$-value } & 0.134 & 0.513 & 0.940 & 0.869 & \\
\hline & SA & & & & \\
\hline SMILE group & $0.081 \pm 0.179$ & $0.223 \pm 0.374^{*}$ & $0.258 \pm 0.452^{*}$ & $0.241 \pm 0.487^{*}$ & 0.467 \\
\hline FS-LASIK group & $0.053 \pm 0.115$ & $0.309 \pm 0.536^{*}$ & $0.270 \pm 0.400^{*}$ & $0.297 \pm 0.388^{*}$ & \\
\hline \multirow[t]{2}{*}{$p_{1}$-value } & 0.338 & 0.316 & 0.887 & 0.503 & \\
\hline & CA & & & & \\
\hline SMILE group & $0.235 \pm 0.167$ & $0.367 \pm 0.349^{*}$ & $0.396 \pm 0.411^{*}$ & $0.407 \pm 0.461^{*}$ & 0.216 \\
\hline FS-LASIK group & $0.265 \pm 0.298$ & $0.571 \pm 0.514^{*}$ & $0.537 \pm 0.435^{*}$ & $0.574 \pm 0.526^{*}$ & \\
\hline \multirow[t]{2}{*}{$p_{1}$-value } & 0.543 & 0.019 & 0.124 & 0.092 & \\
\hline & TA & & & & \\
\hline SMILE group & $0.213 \pm 0.152$ & $0.172 \pm 0.130^{*}$ & $0.163 \pm 0.119^{*}$ & $0.170 \pm 0.172^{*}$ & 0.082 \\
\hline FS-LASIK group & $0.246 \pm 0.200$ & $0.140 \pm 0.090^{*}$ & $0.142 \pm 0.097^{*}$ & $0.130 \pm 0.085^{*}$ & \\
\hline$p_{1}$-value & 0.363 & 0.162 & 0.215 & 0.142 & \\
\hline
\end{tabular}

SD: standard deviation. Preop: preoperative. Post $1 \mathrm{mo}$ : at 1 month postoperatively. Post $6 \mathrm{mo}$ : at 6 months postoperatively. Post $12 \mathrm{mo}$ : at 12 months postoperatively. DA: defocus aberration. AA: astigmatism aberration. SA: spherical aberration. CA: coma aberration. TA: trefoil aberration. $p_{0}=p$ value of the difference for the pre and post-operative variation of the wavefront aberrations between the SMILE and FS-LASIK group. $p_{1}=p$ value of the difference in the wavefront aberrations at each point of time between the SMILE and FS-LASIK groups. When $p<0.05$, the difference was statistically significant. * Compared with that before the surgery, $\mathrm{p}<$ adjusted alpha

whose repeatability had been adequately verified in the previous work [14-16]. Based on the double-pass technique, OQAS is known as the device that can quantitatively provide the data of ocular scatter [27, 28]. The most dominant parameters detected by OQAS include: (1) SR that indicates the convergence ratio of light intensity in the image field of an optical system with aberrations, and the higher the value is, the better the optical quality is; (2) MTF cutoff that characterizes the spatial frequency corresponding to the minimum resolution of human eyes in the modulation transfer function curve, and the higher the value is, the better the optical quality becomes; (3) OSI that objectively reflects the scatter of the refractive medium, and the higher the value is, the muddier the refractive media is. Another evaluation instrument, i.e. iTrace analyzer, is composed of a corneal topographer and an aberrometer whose working principle is ray tracing. It is applicable for the measurement of corneal, internal and total ocular wavefront aberrations [16]. Wavefront aberrations are distortions in the phase of light entering the eye, which leads to the defects in image-forming and thereby decreasing the quality of vision $[29,30]$. They could be caused by the non-optimal surface shapes, irregularities and misalignments in the eye's optical elements. It's generally accepted that as the wavefront aberrations were corrected, the visual sensitivity, night vision, incidence of glare and halo would significantly improve. They could be mathematically represented as the sum of a series of polynomial functions of different orders, and the higher the wavefront aberration is, the more the visual quality is affected. In particular, the high-order aberrations have a greater impact on the visual quality. This study applied iTrace analyzer to measure the total ocular wavefront aberrations before and after the operation, including TWA, TLOA, THOA, etc.

As indicated by the variation of SR, MTF cutoff and OSI in this study, the optical quality descended constantly for one year after both SMILE and FS-LASIK. This finding was different from some previous observations about SMILE surgery [31-33]. Miao et al. used the OQAS to evaluate the optical quality after SMILE and found that the optical quality was not significantly reduced [31]. Niu et al. got a result that for high myopia correction, MTF cutoff declined slightly at 3 months after SMILE but recovered to the preoperative value at the one-year follow-up, and OSI increased at 20 days after surgery but gradually declined to the pre-operative 
level at 3 months [32]. Qin et al. find that no significant difference was found in SR or MTF cut-off in SMILE for high myopia correction before surgery or at any time point after surgery [33]. And few reports were found about the optical quality for high myopia after FS-LASIK evaluated by OQAS. The specific reasons for these differences are unknown, but it was worth the attention of refractive surgeons that there is a decline in the optical quality after the operation.

The results about SR, MTF cutoff and OSI also suggested that SMILE achieved better optical quality than FS-LASIK. In especial, the MTF cutoff at 12 months in SMILE returned to the preoperative level without any difference, possibly indicating the slightly recovery of optical quality as time progressed. Similar research by OQAS was rare between SMILE and FS-LASIK. We considered that the superiority of SMILE here was possibly related to the size of optical zone and high pulse frequency of femtosecond laser. The better optical quality was often accompanied by the larger cutting area in the corneal refractive surgery [34]. It's indicated that SMILE had a comparatively larger effective optical zone than FS-LASIK though a similar optical zone was programmed before the operation $[35,36]$. Besides, the high pulse frequency of femtosecond laser in SMILE is able to alleviate the inflammation reaction and corneal damage, and then induces limited potential loss of the corneal transparency and less scatter when compared with FS-LASIK [5, 37]. Hence, from the perspective of SR, MTF cutoff and OSI, this study suggested that it is more reasonable to have SMILE surgery for the population with high optical quality requirements.

The wavefront aberration provides another objective method to evaluate the postoperative optical quality. In this study, the TWA, TLOA, DA, AA, SA, CA and TA showed a similar increasing or decreasing trends after SMILE and FS-LASIK, except the CA at 1 month postoperatively. And the THOA did not change significantly after SMILE while showed an upward trend after FSLASIK. These results indicated that except the THOAs and CA, SMILE and FS-LASIK had no obvious differences in introducing wavefront aberrations, especially the lower order aberrations. Because the refractive errors were effectively corrected after the two surgeries, the DA and AA decreased obviously. The DA and AA accounted for the majority of the total aberrations, hence the TLOA and TWA also decreased after the operation. As to the higher order aberrations, the increasing trend was similar to the previous studies $[12,38,39]$. Due to the surgery incision and the postoperative wound healing, the asymmetry of eye plane such as irregularity, inclination and decentration might occur, which could be reflected by CAs [24]. Due to the cutting of nerve endings when making the corneal flap in FS-LASIK, the quality of tear film became poor and might also affect the corneal aberrations [40-42]. And a minimal displacement of the corneal flap in FS-LASIK could obviously affect the higher-order aberrations. Besides, SMILE brings less damage to the anterior stromal layer, and the heat load generated by femtosecond laser is lower than that by excimer laser, which both would reduce the influence on central asphericity of cornea [43]. In addition, through the aspheric scanning pattern without scanning interval, SMILE can minimize the higher order aberrations introduced by surgery, while FS-LASIK adopts the excimer laser with scanning interval and would introduce more surgically-induced higher order aberrations [44]. Therefore, there is a disadvantage of FS-LASIK against SMILE in terms of the postoperative higherorder wavefront aberrations. In sum, it proved the advantage of SMILE on the optical quality over FS-LASIK.

Additionally, in this study the change of higher-order aberrations after SMILE or FS-LASIK was not exactly the same with the other researches $[24,45,46]$. It might be related to the different refractive diopters, inspection equipments and assessing parameters, detecting conditions, surgery equipments and procedures, surgeons, etc. For example, by using Sirius System, Jin et al. found that SMILE showed better optical quality than FS-LASIK at larger pupil diameter [13]. Yet no observations of optical quality at different pupil diameters were made in our study. Besides, the results reported in this work cannot represent the general myopic population as the preoperative spherical refraction was from $-6.00 \mathrm{D}$ to $10.00 \mathrm{D}$ and the astigmatism was from $0 \mathrm{D}$ to $-2.50 \mathrm{D}$. We expect more studies to focus on the optical quality of patients with high myopia after different corneal refractive surgeries. And further work is needed to prolong the observation time and increase the sample sizes in order to detect the optical quality more deeply of patients with high myopia after SMILE and FS-LASIK.

\section{Conclusions}

The data reported in this work indicated that both SMILE and FS-LASIK could improve the visual acuity of the patients with high myopia. And the optical quality descended after the operation. The better postoperative visual acuity and refractive results, the higher SR and MTF cutoff, the lower OSI together with the different change of higher order aberrations illustrated that SMILE was superior to FS-LASIK at the correction effect and optical quality for high myopia population. In addition, the combined application of OQAS and iTrace analyzer is a valuable complementary measurement in evaluating the optical quality after the refractive surgery.

\section{Abbreviations}

SMILE: Small incision lenticule extraction; FS-LASIK: Femtosecond laserassisted in situ keratomileusis; UDVA: Uncorrected distance visual acuity; 
CDVA: Corrected distance visual acuity; SE: Spherical equivalent refraction; SR: Strehl ratio; MTF cutoff: Modulation transfer function cutoff frequency; OSI: Objective scatter index; WA: Wavefront aberration; TWA: Total wavefront aberration; TLOA: Total low-order wavefront aberration; THOA: Total highorder wavefront aberration; DA: Defocus aberration; AA: Astigmatism aberration; SA: Spherical aberration; CA: Coma aberration; TA: Trefoil aberration

\section{Acknowledgements}

Not applicable.

\section{Authors' contributions}

The study concept and design were performed by YYW, WXY and WD. The examinations were completed by HT, DKX, HSF and FQM. Data collection and interpretation of data was done by $L Y, X A Q, F Y Y$ and $Z Y$. Writing the manuscript and critical revision of the manuscript were performed by YYW, LYJ and WD. All authors have reviewed the manuscript and approved the final manuscript.

\section{Funding}

This work was supported by the National Natural Science Foundation of China (No.81900890).

\section{Availability of data and materials}

The datasets analysed during the current study are available from the corresponding author on reasonable request.

\section{Declarations}

\section{Ethics approval and consent to participate}

This research was approved by the ethics committee of Xiangya hospital, which followed the tenets of the Declaration of Helsinki. The informed consent was obtained from all the subjects for the operation and the application of data in clinical research. The subjects' personal information was anonymized before the assessment of data.

\section{Consent for publication}

Not applicable.

\section{Competing interests}

We have no any commercial or proprietary interest with the product or company. The authors declare to have no competing interests.

Received: 11 April 2021 Accepted: 9 July 2021

Published online: 02 August 2021

\section{References}

1. Yan H, Gong LY, Huang W, Peng YL. Clinical outcomes of small incision lenticule extraction versus femtosecond laser-assisted LASIK for myopia: a meta-analysis. Int J Ophthalmol. 2017;10(9):1436-45. https://doi.org/10.1824 0/ijo.2017.09.17

2. Tian $Y$, Jiang $H B$, Jiang J, Wen $D$, Xia XB, Song WT. Comparison of implantable collamer lens visian ICL V4 and ICL V4C for high myopia: a cohort study. Medicine. 2017;96(25):e7294. https://doi.org/10.1097/MD. 0000000000007294.

3. Moshirfar M, McCaughey MV, Reinstein DZ, et al. Small-incision lenticule extraction. J Cataract Refract Surg. 2015;41(3):652-65. https://doi.org/10.101 6/j.jcrs.2015.02.006

4. Shen ZR, Shi KD, Yu YH, Yu X, Lin Y, Yao K. Small incision Lenticule extraction (SMILE) versus femtosecond laser-assisted in situ Keratomileusis (FS-LASIK) for myopia: a systematic review and meta-analysis. PLoS One. 2016;11(7):e0158176. https://doi.org/10.1371/journal.pone.0158176.

5. Kunert KS, Blum M, Duncker GIW, Sietmann R, Heichel J. Surface quality of human corneal lenticules after femtosecond laser surgery for myopia comparing different laser parameters. Graefes Arch Clin Exp Ophthalmol. 2011;249(9):1417-24. https://doi.org/10.1007/s00417-010-1578-4.

6. Chen SH, Feng YF, Stojanovic A, Jankov MR II, Wang Q. IntraLase femtosecond laser vs mechanical microkeratomes in LASIK for myopia: a systematic review and meta-analysis. J Refract Surg. 2012;28(1):15-24. https://doi.org/10.3928/1081597X-20111228-02.
7. Fan $L$, Xiong L, Zhang B, Wang Z. Longitudinal and regional non-uniform remodeling of corneal epithelium after topography-guided FS-LASIK. J Refract Surg. 2019;35(2):88-95. https://doi.org/10.3928/1081597X-2019010401.

8. Han T, Zheng K, Chen Y, Gao Y, He L, Zhou X. Four-year observation of predictability and stability of small incision lenticule extraction. BMC Ophthalmol. 2016;16(1):149. https://doi.org/10.1186/s12886-016-0331-0.

9. Chen Y, Xia X. Comparison of the Orbscan II topographer and the iTrace aberrometer for the measurements of keratometry and corneal diameter in myopic patients. BMC Ophthalmol. 2016;16(1):33. https://doi.org/10.1186/s12 886-016-0210-8

10. Zhou JQ, Xu Y, Li MY, et al. Preoperative refraction, age and optical zone as predictors of optical and optical quality after advanced surface ablation in patients with high myopia: a cross-sectional study. BMJ Open. 2018;8: e023877.

11. Gyldenkerne A, Ivarsen A, Hjortdal JO. Comparison of corneal shape changes and aberrations induced by FS-LASIK and SMILE for myopia. J Refract Surg. 2015;31(4):223-9. https://doi.org/10.3928/1081597X-2015030301.

12. Liu ML, Chen YL, Wang DY, Zhou Y, Zhang X, He J, et al. Clinical outcomes after SMILE and femtosecond laser-assisted LASIK for myopia and myopic astigmatism: a prospective randomized comparative study. Cornea. 2016; 35(2):210-6. https://doi.org/10.1097/ICO.0000000000000707.

13. Jin Y, Wang Y, Xu LL, Zuo T, Li H, Dou R, et al. Comparison of the optical quality between small incision Lenticule extraction and femtosecond laser LASIK. J Ophthalmol. 2016;2016:1-9. https://doi.org/10.1155/2016/2507973.

14. Saad A, Saab M, Gatinel D. Repeatability of measurements with a doublepass system. J Cataract Refract Surg. 2010;36(1):28-33. https://doi.org/10.101 6/j.jcrs.2009.07.033

15. Xu Z, Hua YJ, Qiu W, Li G, Wu Q. Precision and agreement of higher order aberrations measured with ray tracing and Hartmann-shack aberrometers. BMC Ophthalmol. 2018;18(1):18. https://doi.org/10.1186/s12886-018-0683-8.

16. Hao J, Li L, Tian F, Zhang H. Comparison of two types of optical quality analyzer for the measurement of high order aberrations. Int J Ophthalmol. 2016;9(2):292-7. https://doi.org/10.18240/ijo.2016.02.22.

17. Xia LK, Ma J, Liu HN, Shi C, Huang Q. Three-year results of small incision lenticule extraction and wavefront-guided femtosecond laser-assisted laser in situ keratomileusis for correction of high myopia and myopic astigmatism. Int J Ophthalmol. 2018;11(3):470-7. https://doi.org/10.18240/ ijo.2018.03.18

18. Yang W, Liu S, Li M, Shen Y, Zhou X. Visual outcomes after small incision Lenticule extraction and femtosecond laser-assisted LASIK for high myopia. Ophthalmic Res. 2020;63(4):427-33. https://doi.org/10.1159/000504304.

19. Han T, Xu Y, Han X, Zeng L, Shang J, Chen X, et al. Three-year outcomes of small incision lenticule extraction (SMILE) and femtosecond laser-assisted laser in situ keratomileusis (FS-LASIK) for myopia and myopic astigmatism. Br J Ophthalmol. 2019:103(4):565-8. https://doi.org/10.1136/bjophthalmol-2 018-312140

20. Wang Y, Ma JN. Future developments in SMILE: higher degree of myopia and hyperopia. Asia Pac J Ophthalmol (Phila). 2019;8(5):412-6. https://doi. org/10.1097/01.APO.0000580128.27272.bb.

21. Blum M, Täubig K, Gruhn C, et al. Five-year results of Small Incision Lenticule Extraction (ReLEx SMILE). Br J Ophthalmol. 2016:100(9):1192.

22. Radhakrishnan H, Allen PM, Charman WN. Dynamics of accommodative facility in myopes. Invest Ophthalmol Vis Sci. 2007:48(9):4375-82. https://doi. org/10.1167/iovs.07-0269.

23. Karimian F, Baradaran-Rafii A, Bagheria A, et al. Accommodative changes after photorefractive keratectomy in myopic eyes. Optometry Vision Sci. 2010;87(11):833-8. https://doi.org/10.1097/OPX.0b013e3181f6fccc.

24. Wang J, Ren YL, Liang K, Jiang Z, Tao L. Changes of corneal high-order aberrations after femtosecond laser-assisted in situ keratomileusis. Medicine 2018;97(18):e0618. https://doi.org/10.1097/MD.0000000000010618.

25. Vilaseca M, Padilla A, Ondategui JC, Arjona M, Güell JL, Pujol J. Effect of laser in situ keratomileusis on vision analyzed using preoperative optical quality. J Cataract Refract Surg. 2010;36(11):1945-53. https://doi.org/10.1016/j.jcrs.201 0.05.029.

26. Lee K, Ahn JM, Kim EK, Kim TI. Comparison of optical quality parameters and ocular aberrations after wavefront-guided laser in-situ keratomileusis versus wavefront-guided laser epithelial keratomileusis for myopia. Graefes Arch Clin Exp Ophthalmol. 2013:251(9):2163-9. https://doi.org/10.1007/s0041 7-013-2356-x. 
27. Artal P, Benito A, Perez GM, et al. An objective scatter index based on double-pass retinal images of a point source to classify cataracts. PLoS One. 2011;6(2):e16823. https://doi.org/10.1371/journal.pone.0016823.

28. Guell JL, Pujol J, Arjona M, et al. Optical quality analysis system: instrument for objective clinical evaluation of ocular optical quality. J Cataract Refract Surg. 2004;30(7):1598-9. https://doi.org/10.1016/j.jcrs.2004.04.031.

29. Mclellan JS, Prieto PM, Marcos S, et al. Effects of interactions among wave aberrations on optical image quality. Vis Res. 2006;46(18):3009-16. https:// doi.org/10.1016/j.visres.2006.03.005.

30. Valentina BS, Ramona B, Speranta S, Calin T. The influence of optical aberrations in refractive surgery. Rom J Ophthalmol. 2015;59(4):217-22.

31. Miao H, He L, Shen Y, Li M, Yu Y, Zhou X. Optical quality and intraocular scattering after femtosecond laser small incision lenticule extraction. J Refract Surg. 2014;30(5):296-302. https://doi.org/10.3928/1081597X-2014041 5-02.

32. Niu L, Miao H, Tian M, Fu D, Wang X, Zhou X. One year visual outcomes and optical quality of femtosecond laser small incision lenticule extraction and Visian implantable Collamer Lens (ICL V4C) implantation for high myopia. Acta Ophthalmol. 2020;98(6):e662-7. https://doi.org/10.1111/a os.14344.

33. Qin Q, Bao L, Yang L, He Z, Huang Z. Comparison of visual quality after EVO-ICL implantation and SMILE to select the appropriate surgical method for high myopia. BMC Ophthalmol. 2019;19(1):21. https://doi.org/10.1186/s12 886-019-1029-x

34. Alarcón A, Rubiño M, PérezOcón F, et al. Theoretical analysis of the effect of pupil size, initial myopic level, and optical zone on quality of vision after corneal refractive surgery. J Refract Surg. 2012;28(12):901-6. https://doi.org/1 0.3928/1081597X-20121106-01.

35. Damgaard I, Ang M, Mahmoud A, et al. Functional optical zone and centration following SMILE and LASIK: a prospective, randomized, contralateral eye study. J Refract Surg. 2019;35(4):230-7. https://doi.org/10.3 928/1081597X-20190313-01.

36. Hou J, Wang Y, Lei Y, Zheng X. Comparison of effective optical zone after small-incision lenticule extraction and femtosecond laser-assisted laser in situ keratomileusis for myopia. J Cataract Refract Surg. 2018;44(10):1179-85. https://doi.org/10.1016/j.jcrs.2018.06.046.

37. Denoyer A, Landman E, Trinh L, Faure JF, Auclin F, Baudouin C. Dry eye disease after refractive surgery comparative outcomes of small incision Lenticule extraction versus LASIK. Ophthalmology. 2015;122(4):669-76. https://doi.org/10.1016/j.ophtha.2014.10.004.

38. Zhang H, Wang Y, Li H. Corneal spherical aberration and corneal Asphericity after small incision Lenticule extraction and femtosecond laser-assisted LASI K. J Ophthalmol. 2017;2017:4921090.

39. Qian YS, Chen X, Naidu RK, Zhou X. Comparison of efficacy and visual outcomes after SMILE and FS-LASIK for the correction of high myopia with the sum of myopia and astigmatism from -10.00 to -14.00 dioptres. Acta Ophthalmol. 2020:98(2):e161-72. https://doi.org/10.1111/aos.14078.

40. Montes-Mico R. Role of the tear film in the optical quality of the human eye. J Cataract Refract Surg. 2007;33(9):1631-5. https://doi.org/10.1016/j.jcrs.2 007.06.019.

41. Benito A, Perez GM, Mirabet S, et al. Objective optical assessment of tearfilm quality dynamics in normal and mildly symptomatic dry eyes. J Cataract Refract Surg. 2011:37(8):1481-7. https://doi.org/10.1016/j.jcrs.2011.03.036.

42. Serrao S, Buratto L, Lombardo G, de Santo MP, Ducoli P, Lombardo M. Optimal parameters to improve the interface quality of the flap bed in femtosecond laser-assisted laser in situ keratomileusis. J Cataract Refract Surg. 2012;38(8):1453-9. https://doi.org/10.1016/j.jcrs.2012.05.021.

43. George MR, Shah RA, Hood C, Krueger RR. Transitioning to optimized correction with the WaveLight ALLEGRETTO WAVE: case distribution, visual outcomes, and wavefront aberrations. J Refract Surg. 2010;26(10):S806-13. https://doi.org/10.3928/1081597X-20100921-07.

44. Lin F, XU Y, Yang Y. Comparison of the visual results after SMILE and femtosecond laser-assisted LASIK for myopia. J Refract Surg. 2014;30(4):24854. https://doi.org/10.3928/1081597X-20140320-03.

45. Jin HY, Wan T, Wu F, et al. Comparison of visual results and higher-order aberrations after small incision lenticule extraction (SMILE): high myopia vs. mild to moderate myopia. BMC Ophthalmol. 2017;17(1):118.

46. Jaurrieta-Hinojos JN, Fernandez $\mathrm{O}$. Changes in visual acuity and high-order aberrations after SMILE. Invest Ophthalmol Vis Sci. 2016;57(12):4884.

\section{Publisher's Note}

Springer Nature remains neutral with regard to jurisdictional claims in published maps and institutional affiliations.
Ready to submit your research? Choose BMC and benefit from:

- fast, convenient online submission

- thorough peer review by experienced researchers in your field

- rapid publication on acceptance

- support for research data, including large and complex data types

- gold Open Access which fosters wider collaboration and increased citations

- maximum visibility for your research: over $100 \mathrm{M}$ website views per year

At BMC, research is always in progress.

Learn more biomedcentral.com/submissions 